\title{
Lateral Orbitofrontal Artery
}

National Cancer Institute

\section{Source}

National Cancer Institute. Lateral Orbitofrontal Artery. NCI Thesaurus. Code C32943.

An artery branching from the extremity of the middle cerebral artery that supplies the lateral cortex and inferior frontal lobe. 